\title{
Map Analysis of the Progress of Tourism Interpretation Research: Based on the Measurement Comparison of Chinese and International Literature
}

\author{
Jia Li \\ School of Tourism Management, Sanya College, 572000, Sanya, China
}

\begin{abstract}
This paper collects research literature on tourism interpretation at home and abroad, uses Citespace software to analyze the co-linearity of keyword networks, studies the thematic collinear map, compares the content of collinear maps of the past years, and finds that the theoretical basis of domestic research is relatively simple, especially there is no complete and systematic research system on the interpretation of national parks. In addition, the sources of domestic research funds are mostly concentrated in the National Natural Science Foundation, which means that regional governments and local industry organizations pay insufficient attention to research in this field, and the research results have little guiding effect on practice. Studying the scientific explanation of tourism, excavating the natural and humanistic knowledge of tourist attractions, and presenting them in an effective and reasonable way can solve the problem of low quality of tourism supply and respond to the reform of the supply side of China's tourism economy. To this end, this study will lay the foundation for the improvement of the quality of domestic tourism service supply.
\end{abstract}

\section{Introduction}

The study of tourism interpretation began when Tilden first gave a complete and scientific definition of interpretation in the creation of a national park. Interpretation is not just a one-way communication, but a service function provided by national parks and municipal parks[1], which expresses natural science knowledge in easy-to-understand language. In the natural environment, people's awareness of the environment, surrounding things and the events associated with them are created through the explanation, thus producing pleasant communication and enriching people's lives and experiences. In the scholars' definition of tourism interpretation, tourist's intelligence, cognition, experience and emotion are emphasized and the process of interpretation requires knowledge transmission employing correct communication methods and tools to give visitors a meaningful experience-----an emotional association between a person and nature. However, China's current tourism interpretation cannot achieve such a goal. There are many tourists in China, and most tourism activities are mainly mass sight-seeing. The peaks of holiday or holiday in winter and summer vacation are overcrowded. The interpretations of cultural landscape with rich historical and cultural connotations or the unique natural scenery of world heritage are too shallow. Intellectual information and knowledge content is vague, unclear, and even interpret natural cultural heritage with non-scientific myths and legends. It cannot enrich the tourist experience and establish a meaningful emotional connection between man and nature. People's expectations for a better experience are far greater than the reality experience, resulting in a mismatch between demand and supply, restricting the vigorous development of China's tourism market. Domestic research on interpretation is lagging behind and cannot guide existing social practice. The research of interpretation originates from national park explanation, having a very complete body of knowledge including the concept, process and purpose of interpretation, the way and method of explanation, the medium of explanation, the explanation plan and explanation system. The article uses Citespace5.3 software to compare Chinese studies with international scholars' research in the field of tourism interpretation. It is expected to find gaps and deficiencies, guide and standardize the tourism explanations of Chinese domestic attractions, with a view to improving the quality of overall tourism supply services and helping the development of China's tourism market.

\section{Tourism commentary keyword collinear analysis}

This study uses the "Tourism Interpretation" and "Legacy Interpretation" as keywords to retrieve in Chinese Knowledge Network, Elsevier, Springer, and Web of science, 531 Chinese documents and 314 foreign language documents are searched and analyzed by 
CiteSpace software respectively. The high-frequency keywords in foreign research (according to frequency, vocabulary threshold $\geq 2$ ) are heritage interpretation, environmental commentary, tourist behavior, geological tourism, tourist satisfaction, geological protection, knowledge learning, sustainable tourism. The high-frequency keywords appearing in domestic research (according to the frequency, the threshold of $\geq 2$ ) are the explanation system, tourism explanation system, tourism explanation, environmental explanation, geological park, forest park, tourist scenic spot and environmental education. The frequency of research selection is more than 5 times for co-occurrence analysis. According to the frequency of co-occurrence relations, foreign research fields focus on the behavior of tourists, the satisfaction of tourists, the environmental learning and environmental education in the process of interpretation of the heritage, and the interpretation of cultural heritage, the knowledge learning of tourists, the explanation of geological tourism and sustainable development are the main points during the explanation process.

The focus of domestic research is on geologic park interpretation, forest park interpretation, tourism (scenic) interpretation system, environmental explanation and environmental education, interpretation perception, satisfaction, interpretation system construction and optimization. The co-occurrence network map is not completely "scattered", relatively showing a "mesh" structure, indicating that the research is systematic, but the research results are not particularly rich (see Figure $1)$.

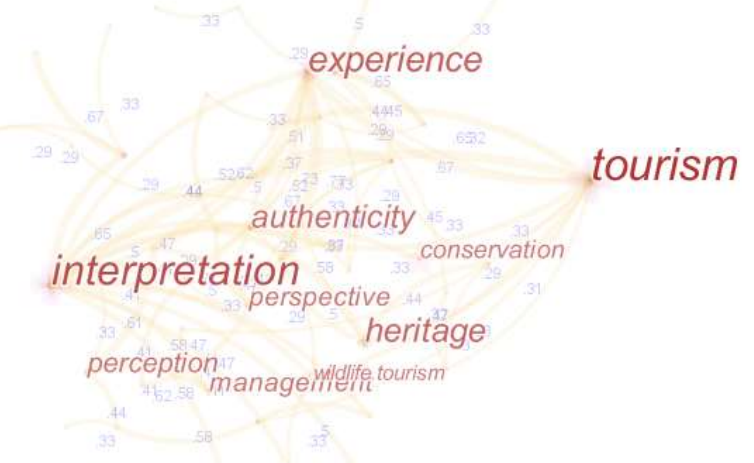

Figure 1. Map of international tourism interpretation research.

\section{Annual research map comparative analysis}

The comparison of the thematic maps over the years shows that the concept of "interpretation" began in the early 20th century when scholars studied European and American national parks. In the 1960s, the definition and system framework of tourism interpretation were further developed[2]. by the 1990 s, foreign scholars began to pay attention to the media. They generally believed that the application of the complete multi-media system could obtain the best interpretation effect[3]. In 1999, the domestic research on the tourism interpretation was just at the beginning [4], it has been in the stage of introducing the basic theory of foreign explanations studies. The specific content involves the concept, planning and framework of the interpretation system[5-6].

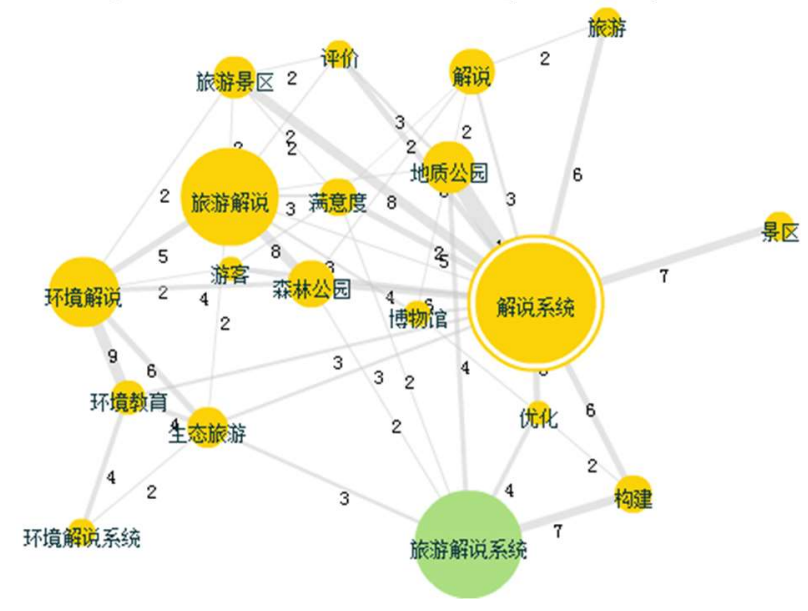

Figure 2. Map of Chinese tourism interpretation research.

By the beginning of the 21 st century, with the deepening and development of tourism experience, foreign scholars began to pay attention to emerging tourism types and multidisciplinary research perspectives, and the research on tourism commentary was more abundant[7]. Changes in the thematic maps show that before 2013, foreign research focused on the analysis of the coupling relationship between demand and satisfaction from the psychological factors of tourists[8]. After 2013, scholars mainly focused on explaining how interpretation impact environmental behavior and environmental learning[9-10], and how the different values of visitors impact the environmental education interpretation[11], and geological tourism, dark tourism. It can be seen that after 2013 the research content is more detailed, and more concerned with the educational impact on people. Domestic research showed an turning point of research trends in 2014. From 2000 to 2014, research focused on the construction, interpretation quality and effect evaluation of the interpretation system. In 2014 and after, the domestic research began to pay attention to the interpretation system of national parks, geological parks and forest parks, and focused on the geological parks and forest parks in the past one or two years. The research based on geological parks and forest parks, and the content is richer and deeper, specifically related to the tourist experience of the interpretation system of natural and cultural heritage, the relationship between commentary and tourist loyalty, environmental commentary and environmental education, and sustainable tourism Development[12]. The domestic research on interpretation has gone through a preliminary period and has just entered a period of rapid development. To sum up, the domestic research takes the study results of other countries for reference and follows the research trend abroad without too much innovation in both the basic theory research and study methods. It is also short of the perspective of multi-disciplinary research. More researches focus on single scenic area from microcosmic aspects and lack of applicability. Domestic 
tourism interpretation research is still weak, far behind the booming practice.

\section{Comparison analysis of research areas}

Foreign research on tourism interpretation focuses on social science, leisure hospitality business, economic green and sustainable development, science and technology, science and technology, sociology and management (see figure 3). Research foundations include National Natural Science Foundation of China, National Science Foundation of China, Netherlands Organization for Scientific Research (NWO), Province of Fryslan, Qatar University, Fulbright Fellowship Award, National Environmental Research Program, Hong Kong Polytechnic University, Centre for Research and Studies, Department of Science and Innovation of Spanish government and ect.

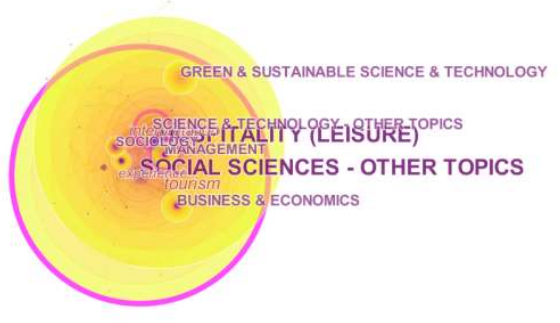

Figure 3. International research field.

The domestic research area focuses on tourism, architecture, science and engineering, foreign language, literature, culture, macroeconomic management and sustainable development, environmental science and resource utilization, Chinese language and script resources, forestry, archaeology, agriculture, natural geography and surveying and mapping. Research fund includes national natural science fund, national social science fund, national science and technology support program, education commission of Hunan province, education commission of Shanxi province, soft science research program of Hunan province, soft science research program of Hunan province (see figure 4).

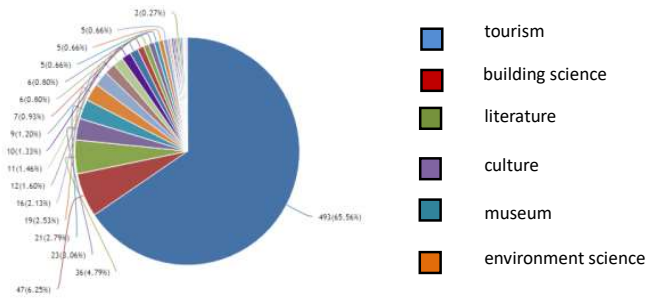

Figure 4. Domestic research field.

To sum up, from the perspective of research field abroad, the research in this field involves multi-disciplines and cross-fields such as social science, natural science and management science, while the research in this field of China is mainly based on tourism, with narrow theoretical background and insufficient perspective. From the source of funds, foreign support is extensive, both academic funds and government and local support. Research from the national natural science foundation accounts for more than half of the total research. It can be seen that China regards this research as academic research, which has not been paid attention and utilized by the government and other local industries.

\section{Opinions and conclusions}

\subsection{Opinions}

The study of scientific interpretation, the exploration of the natural and humanistic knowledge of tourist destinations and the display of it by effective means can solve the problem of low quality of tourism supply and respond to the supply-side reform of tourism economy. Scientific knowledge into the explanation content, participation form and the design of link of education, will upset the blundering, fast way of travel, change condition of lacking of meaning and connotation tourism, making travel "slow" down, "pure" experience, "rich" observation and recreation, can better stimulate domestic demand.

\subsection{Conclusions}

Through the collection and analysis of domestic and foreign literature related to tourism interpretation, this article shows that the research foundation of foreign countries is not limited to the field of tourism, and the theoretical and knowledge background is rich, and it has gained extensive social support and recognition, especially the interpretation of national parks and the impact of interpretation on the environment. The theoretical basis of domestic research is relatively single, and there is no complete and systematic concept of national park in China, and the cognition of interpretation is mostly limited to tour guide's explanation, which restricts the development and the function of interpretation.

In general, the research on interpretation originates from European and American national parks and also develops in European and American national parks studies. In particular, American national parks have rich practice achievements in interpretation knowledge presentation, project design, environment education for teenagers and popular science education for geological knowledge. The study of domestic tourism interpretation started late, and the theory lags far behind the booming practice. The study of domestic tourism interpretation, especially in terms of how to guide the practice, needs to refer to the classic cases of foreign countries. The tourism environment is rich in content, and the backgrounds of the tourists with different preferences are diverse. The tourism experience emphasizes the real-time experience of tourists in the natural scene, so it is necessary to use place dependence, emotional intelligence, The knowledge of sociology and psychology such as cognition within the group to construct and evaluate the interpretation system. 
Therefore, the interpretation of tourism needs the in-depth elaboration of multidisciplinary theory.

\section{References}

1. Tilden F, Craig R B. Chapel Hill: The University of North Carolina Press, 25-35, (2009)

2. Wagar A J. Journal of Interpretation, 1, 1-8, (1976)

3. Istvan L B. Communicating the arcane: A conceptual framework for environmental interpretation [D]. Ph.D. Dissertation, University of Washington, 32-45, (1993)

4. Wu Bi-Hu, Jin Hua, Zhang Li. Journal of tourism Tribune, 14, 27-29. (1999)

5. Tang gala. Journal of tourism Tribune, 18, 14-17(2003)
6. Xinjian Li. Human geography, 19, 43-46(2004)

7. Cohen H E, Ifergan M, Cohen E. Annals of Tourism Research, 29, 919-932.(2002)

8. Wight C A, Lennon J J. Tourism Management, 28, 519-529(2007)

9. Ballantyne R, Packer J. Tourism Management, 32, 770-779(2011)

10. Siu Y N, Zhang J T, et al.. Tourism Management, 36, 293-303.(2013)

11. Ballantyne, R., Packer, J., Hughes, K.,\& Gill, C. Post-visit reinforcement of zoo conservation message: The design and testing of an action resource website. (2017) (to be published)

12. Tao Wei, Du Xiaofang. Journal of tourism Tribune, 8 , 47-52(2009) 